\title{
NONLINEAR ITERATION SOLUTION FOR THE FULL GLUON PROPAGATOR AS A FUNCTION OF THE MASS GAP
}

\author{
V. Gogokhia* \\ HAS, CRIP, RMKI, Depart. Theor. Phys., Budapest 114, P.O.B. 49, H-1525, Hungary
}

(Dated: April 22, 2022)

\begin{abstract}
We have explicitly shown that QCD is the color gauge invariant theory at non-zero mass gap as well. It has been defined as the value of the regularized full gluon self-energy at some finite point. The mass gap is mainly generated by the nonlinear interaction of massless gluon modes. All this allows one to establish the structure of the full gluon propagator in the explicit presence of the mass gap. In this case, the two independent general types of formal solutions for the full gluon propagator as a function of the regularized mass gap have been found. The nonlinear iteration solution at which the gluons remain massless is explicitly present. The existence of the solution with an effective gluon mass is also demonstrated.
\end{abstract}

PACS numbers: $11.15 . \mathrm{Tk}, 12.38 . \mathrm{Lg}$

\section{INTRODUCTION}

Quantum Chromodynamics (QCD) [1, 2] is widely accepted as a realistic quantum field gauge theory of the strong interactions not only at the fundamental (microscopic) quark-gluon level but at the hadronic (macroscopic) level as well. This means that in principle it should describe the properties of experimentally observed hadrons in terms of experimentally never seen colored quarks and gluons (the color confinement phenomenon), i.e., to describe the hadronic world from first principles - an ultimate goal of any fundamental theory.

The Lagrangian of QCD, however, does not contain explicitly any of the mass scale parameters which could have a physical meaning even after the corresponding renormalization program is performed. This clearly shows that it is not enough to know it in order to calculate the physical observables in low-energy QCD from first principles. It is also important to know the true dynamical structure of the QCD ground state especially at large distances, which may be source of the above-mentioned mass scale parameter. If it will survive the renormalization program, then QCD is a complete and self-consistent theory without the need to introduce some extra degrees of freedom in order to generate it. In this way it may become a mass gap so needed in non-perturbative (NP) QCD in order to explain the above-mentioned color confinement and other NP effects [3]. It will be responsible for the NP QCD dynamics as $\Lambda_{Q C D}$ is responsible for the nontrivial perturbative QCD dynamics (scale violation, asymptotic freedom (AF) [1, 2]).

The propagation of gluons is one of the main dynamical effects in the QCD vacuum. In our previous work [4] it has been shown that the only place when the mass gap may appear is the corresponding Schwinger-Dyson (SD) equation of motion for the full gluon propagator. It should be complemented by the corresponding Slavnov-Taylor (ST) identity (see next section). The importance of this equation is due to the fact that its solutions reflect the quantum-dynamical structure of the QCD ground state. It is highly nonlinear (NL) equation, and therefore the number of independent solutions, which should be considered on equal footing, is not fixed a priori. The color gauge structure of this equation has been investigated in detail in the above-mentioned paper [4]. We have explicitly shown that the color gauge invariance of QCD is consistent with the mass gap, generated in the gluon sector of QCD.

Our primary goal in this investigation is to find formal solutions for the full gluon propagator as a function of the regularized mass gap. However, for the sake of completeness and further clarity, it is instructive to describe briefly the main results of Ref. [4] in the subsequent section.

\section{THE COLOR GAUGE INVARIANCE OF QCD AT NON-ZERO MASS GAP}

QCD is a $S U(3)$ color gauge invariant theory. As underlined above, its dynamical context is determined by the corresponding equations of motion, among which the SD equation for the full gluon propagator plays an important role. It can be written as follows:

*gogohia@rmki.kfki.hu 


$$
D_{\mu \nu}(q)=D_{\mu \nu}^{0}(q)+D_{\mu \rho}^{0}(q) i \Pi_{\rho \sigma}(q ; D) D_{\sigma \nu}(q)
$$

where

$$
D_{\mu \nu}^{0}(q)=i\left\{T_{\mu \nu}(q)+\xi L_{\mu \nu}(q)\right\} \frac{1}{q^{2}}
$$

is the free gluon propagator, and $\xi$ is the gauge-fixing parameter. Also, here and everywhere below $T_{\mu \nu}(q)=\delta_{\mu \nu}-$ $\left(q_{\mu} q_{\nu} / q^{2}\right)=\delta_{\mu \nu}-L_{\mu \nu}(q)$, as usual. $\Pi_{\rho \sigma}(q ; D)$ is the full gluon self-energy which depends on the full gluon propagator due to the non-abelian character of QCD. Thus the gluon SD equation is highly NL one. Evidently, we omit the color group indices, since for the gluon propagator (and hence for its self-energy) they factorize, for example $D_{\mu \nu}^{a b}(q)=$ $D_{\mu \nu}(q) \delta^{a b}$. Diagrammatic representation of the gluon SD equation (2.1) is shown in our previous work [4], as well as the detail description of the full gluon self-energy $\Pi_{\rho \sigma}(q ; D)$. It is the sum of a few terms which are tensors, having the dimensions of mass squared. All these skeleton loop integrals are therefore quadratically divergent in perturbation theory (PT), and so they are assumed to be regularized, as discussed below. Let us note in advance that here and below the signature is Euclidean, since it implies $q_{i} \rightarrow 0$ when $q^{2} \rightarrow 0$ and vice-versa.

\section{A. The mass gap}

Let us introduce the general mass scale parameter $\Delta^{2}(D)$, having the dimensions of mass squared, by the subtraction from the full gluon self-energy its value at $q=0$. Thus, one obtains

$$
\Pi_{\rho \sigma}^{s}(q ; D)=\Pi_{\rho \sigma}(q ; D)-\Pi_{\rho \sigma}(0 ; D)=\Pi_{\rho \sigma}(q ; D)-\delta_{\rho \sigma} \Delta^{2}(D)
$$

which is nothing but the definition of the subtracted full gluon self-energy $\Pi_{\rho \sigma}^{s}(q ; D)$. Contrary to QED, QCD being a non-abelian gauge theory can suffer from infrared (IR) singularities in the $q^{2} \rightarrow 0$ limit due to the self-interaction of massless gluon modes. Thus the initial subtraction at zero in the definition (2.3) may be dangerous [1]. That is why in all the quantities below the dependence on the finite (slightly different from zero) dimensionless subtraction point $\alpha$ is to be understood. From a technical point of view, however, it is convenient to put formally $\alpha=0$ in all the derivations below, and to restore the explicit dependence on non-zero $\alpha$ in all the quantities only at the final stage. At the same time, in all the quantities where the dependence on $\lambda$ (which is the dimensionless ultraviolet (UV) regulating parameter) and $\alpha$ is not shown explicitly, nevertheless, it should be assumed. For example, $\Delta^{2}(D) \equiv \Delta^{2}(\lambda, \alpha ; D)$ and similarly for all other quantities. So all the expressions are regularized. For our purpose, in principle, it is not important how $\lambda$ and $\alpha$ have been introduced. They should be removed at the final stage only as a result of the renormalization program.

By the mass gap we understand some fixed mass squared which is related to $\Delta^{2}(D)$ as follows:

$$
\Delta^{2}(D)=\Delta^{2} \times c(D)
$$

where the dimensionless constant $c(D)$ depends on $D$, while the fixed mass squared $\Delta^{2}$ does not depend on $D$. It will be called the mass gap. As the general mass scale parameter itself and constant $c(D)$, it may depend on all other dimensionless parameters of the theory, namely $\Delta^{2} \equiv \Delta^{2}\left(\lambda, \alpha, \xi, g^{2}\right)$, where $g^{2}$ is the coupling constant squared and so on. In this section we will not distinguish between $\Delta^{2}(D)$ and $\Delta^{2}$, calling both the mass gap, for simplicity. From the subtraction (2.3) it follows that the mass gap $\Delta^{2}$, having the dimensions of mass squared, is dynamically generated in the QCD gluon sector. It is defined as the value of the full gluon self-energy at some finite point (see discussion above). It is mainly due to the nonlinear interaction of massless gluon modes. Let us remind that no truncations/approximations/assumptions/, as well as no special gauge choice are made for the regularized skeleton loop integrals, contributing to the full gluon self-energy [4].

\section{B. The transversality of the full gluon self-energy}

Let us continue with the general decompositions of the full gluon self-energy and its subtracted counterpart, which enter the subtraction (2.3), as follows: 


$$
\begin{aligned}
& \Pi_{\rho \sigma}(q ; D)=T_{\rho \sigma}(q) q^{2} \Pi\left(q^{2} ; D\right)+q_{\rho} q_{\sigma} \tilde{\Pi}\left(q^{2} ; D\right), \\
& \Pi_{\rho \sigma}^{s}(q ; D)=T_{\rho \sigma}(q) q^{2} \Pi^{s}\left(q^{2} ; D\right)+q_{\rho} q_{\sigma} \tilde{\Pi}^{s}\left(q^{2} ; D\right),
\end{aligned}
$$

where all the invariant functions of $q^{2}$ are dimensionless ones, while in addition the invariant functions $\Pi^{s}\left(q^{2} ; D\right)$ and $\tilde{\Pi}^{s}\left(q^{2} ; D\right)$ cannot have the pole-type singularities in the $q^{2} \rightarrow 0$ limit, since $\Pi_{\rho \sigma}^{s}(0 ; D)=0$, by definition; otherwise they remain arbitrary.

Contracting them with $q_{\rho}$ along with the subtraction (2.3), one obtains

$$
\tilde{\Pi}\left(q^{2} ; D\right)=\tilde{\Pi}^{s}\left(q^{2} ; D\right)+\frac{\Delta^{2}(D)}{q^{2}},
$$

and

$$
\Pi\left(q^{2} ; D\right)=\Pi^{s}\left(q^{2} ; D\right)+\frac{\Delta^{2}(D)}{q^{2}} .
$$

It is worth emphasizing that the full gluon self-energy has a massless single particle singularity due to non-zero mass gap $\Delta^{2}(D)$, which is of the non-perturbative (NP) origin. At the same time, its subtracted counterpart cannot have such a singularity, as mentioned above. In other words, this means that in the explicit presence of the mass gap both invariant functions of the full gluon self-energy gain additional contributions due to it (of course, not only at some finite subtraction point $q^{2}=\mu^{2} \neq 0$ ). If the mass gap is welcome in the transversal invariant function $\Pi\left(q^{2} ; D\right)$, it is not welcome in its longitudinal counterpart $\tilde{\Pi}\left(q^{2} ; D\right)$, since just it violates the ST identity. Let us also note in advance that transversality of the full gluon self-energy and its subtracted counterpart can be achieved only in the formal $\Delta^{2}(D)=0$ limit (for a brief discussion of all these preliminary remarks see subsections below). So in the general case of non-zero $\Delta^{2}(D)$ only two possibilities remain.

(i). Both are not transversal and then

$$
\begin{aligned}
& q_{\rho} \Pi_{\rho \sigma}(q ; D)=q_{\sigma} q^{2} \tilde{\Pi}\left(q^{2} ; D\right)=q_{\sigma}\left[q^{2} \tilde{\Pi}^{s}\left(q^{2} ; D\right)+\Delta^{2}(D)\right] \neq 0, \\
& q_{\rho} \Pi_{\rho \sigma}^{s}(q ; D)=q_{\sigma} q^{2} \tilde{\Pi}^{s}\left(q^{2} ; D\right)=q_{\sigma}\left[q^{2} \tilde{\Pi}\left(q^{2} ; D\right)-\Delta^{2}(D)\right] .
\end{aligned}
$$

The last inequality in the first of the relations $(2.8)$ follows from the fact that $\tilde{\Pi}^{s}\left(q^{2} ; D\right)$ cannot have a single particle singularity $-\Delta^{2}(D) / q^{2}$ in order to cancel $\Delta^{2}(D)$.

(ii). Transversality of the subtracted gluon self-energy is maintained, i.e., $\tilde{\Pi}^{s}\left(q^{2} ; D\right)=0$ and then

$$
q_{\rho} \Pi_{\rho \sigma}(q ; D)=q_{\sigma} q^{2} \tilde{\Pi}\left(q^{2} ; D\right)=q_{\sigma} \Delta^{2}(D) \neq 0, \quad q_{\rho} \Pi_{\rho \sigma}^{s}(q ; D)=0,
$$

Contrary to the first case, now we know how precisely the transversality of the full gluon self-energy is violated. So it is always violated at non-zero mass scale parameter $\Delta^{2}(D)$. In this connection one thing should be made perfectly clear. It is the initial subtraction (2.3) which leaves the subtracted gluon-self energy logarithmical divergent only, and hence the invariant function $\Pi^{s}\left(q^{2} ; D\right)$ is free of the quadratic divergences, but a logarithmic ones can be still present in it, at any $D$. Since the transversality condition for the full gluon self-energy is violated in these relations, that is why we cannot disregard $\Delta^{2}(D)$ from the very beginning (compare with the pure quark case considered in our initial work [4]).

\section{The ST identity for the full gluon propagator}

In order to calculate the physical observables in QCD from first principles, we need the full gluon propagator rather than the full gluon self-energy. The basic relation to which the full gluon propagator should satisfy is the corresponding ST identity

$$
q_{\mu} q_{\nu} D_{\mu \nu}(q)=i \xi .
$$


It is a consequence of the color gauge invarince/symmetry of QCD, and therefore "is an exact constraint on any solution to QCD" [1]. This is true for any other ST identities. Being a result of this exact symmetry, it is the general one, and it is important for the renormalization of QCD. If some equation, relation or the regularization scheme, etc. do not satisfy it automatically, i.e., without any additional conditions, then they should be modified and not this identity (identity is an equality, where both sides are the same, i.e., there is no room for additional conditions). In other words, all the relations, equations, regularization schemes, etc. should be adjusted to it and not vice versa. It implies that the general tensor decomposition of the full gluon propagator is

$$
D_{\mu \nu}(q)=i\left\{T_{\mu \nu}(q) d\left(q^{2}\right)+\xi L_{\mu \nu}(q)\right\} \frac{1}{q^{2}},
$$

where the invariant function $d\left(q^{2}\right)$ is the corresponding Lorentz structure of the full gluon propagator (sometimes we will call it as the full effective charge ("running"), for simplicity). Let us emphasize once more that these basic relations are to be satisfied in any case, for example, whether the mass gap or any other mass scale parameter is put formally zero or not.

On account of the exact relations (2.5), (2.6) and (2.7), the initial gluon SD equation (2.1) can be equivalently re-written down as follows:

$$
D_{\mu \nu}(q)=D_{\mu \nu}^{0}(q)+D_{\mu \rho}^{0}(q) i T_{\rho \sigma}(q)\left[q^{2} \Pi^{s}\left(q^{2} ; D\right)+\Delta^{2}(D)\right] D_{\sigma \nu}(q)+D_{\mu \rho}^{0}(q) i L_{\rho \sigma}(q) q^{2} \tilde{\Pi}\left(q^{2} ; D\right) D_{\sigma \nu}(q)
$$

Contracting this equation with $q_{\mu}$ and $q_{\nu}$, one arrives at $q_{\mu} q_{\nu} D_{\mu \nu}(q)=i \xi-i \xi^{2} \tilde{\Pi}\left(q^{2} ; D\right)$, so the ST identity $(2.10)$ is not automatically satisfied. In order to get from this relation the ST identity, one needs to put $\tilde{\Pi}\left(q^{2} ; D\right)=0$, which is equivalent to $\tilde{\Pi}^{s}\left(q^{2} ; D\right)=-\left(\Delta^{2}(D) / q^{2}\right)$, as it follows from the relation (2.6). This, however, is impossible since $\tilde{\Pi}^{s}\left(q^{2} ; D\right)$ cannot have the power-type singularities at small $q^{2}$, as underlined above. The only solution to the previous relation is to disregard $\Delta^{2}(D)$ from the very beginning, i.e., put formally zero $\Delta^{2}(D)=0$ everywhere. In this case from all the relations it follows that the gluon full self-energy coincides with its subtracted counterpart, and both quantities become purely transversal, i.e., $\Pi\left(q^{2} ; D\right)=\Pi^{s}\left(q^{2} ; D\right)$ and $\tilde{\Pi}\left(q^{2} ; D\right)=\tilde{\Pi}^{s}\left(q^{2} ; D\right)=0$ (see relations $(2.5)-(2.7))$.

The one way to satisfy the ST identity and thus to maintain the color gauge structure of QCD is to discard the mass gap $\Delta^{2}(D)$ from the very beginning, i.e., put it formally zero $\Delta^{2}(D)=0$ in all the equations, relations, etc. In this limit the initial gluon SD equation (2.12) is modified to

$$
D_{\mu \nu}^{P T}(q)=D_{\mu \nu}^{0}(q)+D_{\mu \rho}^{0}(q) i T_{\rho \sigma}(q) q^{2} \Pi^{s}\left(q^{2} ; D^{P T}\right) D_{\sigma \nu}^{P T}(q)
$$

and the corresponding Lorentz structure which appears in Eq. (2.11) becomes

$$
d^{P T}\left(q^{2}\right)=\frac{1}{1+\Pi^{s}\left(q^{2} ; D^{P T}\right)} .
$$

It is easy to see that the gluon SD equation (2.13) automatically satisfies the ST identity (2.10) now. Evidently, in the formal $\Delta^{2}(D)=0$ limit we denote $D_{\mu \nu}(q)$ and $d\left(q^{2}\right)$ as $D_{\mu \nu}^{P T}(q)$ and $d^{P T}\left(q^{2}\right)$, respectively (for reason see below). As it has been pointed out in Ref. [4], in this case there will be no problems for ghosts to accomplish their role, namely to cancel the longitudinal component in the full gluon propagator $(2.13)$.

\section{The general structure of the full gluon propagator}

The formal $\Delta^{2}(D)=0$ limit is a real way how to preserve the color gauge invariance in QCD. Then a natural question arises why does the mass gap $\Delta^{2}(D)$ exist in this theory at all? There is no doubt that the color gauge invariance of QCD should be maintained at non-zero mass gap as well, since it is explicitly present in the full gluon self-energy, and hence in the full gluon propagator. However, by keeping it "alive", the two important problems arise. The first problem is how to replace the original gluon SD equation (2.12), since it is not consistent with the ST identity unless the mass gap is discarded from the very beginning (see above). The second problem is how to make the full gluon propagator purely transversal when the mass gap is explicitly present.

By introducing the spurious technics we were able to show that the ST identity (2.10) can be automatically satisfied at non-zero mass gap $\Delta^{2}(D)$ as well. In other words, our aim is to save the mass gap in the transversal invariant 
function (2.7), while removing it from the longitudinal invariant function (2.6), but without going formally to the PT $\Delta^{2}=0$ limit. In order to keep the mass gap "alive", and, at the same time, to satisfy the ST identity (2.10), we introduced a temporary dependence on $\Delta^{2}(D)$ in the free gluon propagator, thus making it an auxiliary (spurious) free gluon propagator. Substituting it into the initial gluon SD equation (2.12) and restoring again the dependence on the free gluon propagator, such obtained gluon SD equation should satisfy the ST identity (2.10). After doing some tedious algebra, one finally obtains [4]

$$
D_{\mu \nu}(q)=D_{\mu \nu}^{0}(q)+D_{\mu \rho}^{0}(q) i T_{\rho \sigma}(q)\left[q^{2} \Pi^{s}\left(q^{2} ; D\right)+\Delta^{2}(D)\right] D_{\sigma \nu}(q)
$$

Such modified gluon SD equation (2.15) is satisfied by the same expression for the Lorentz structure $d\left(q^{2}\right)$ in Eq. $(2.11)$ as the original gluon SD equation (2.12), namely

$$
d\left(q^{2}\right)=\frac{1}{1+\Pi^{s}\left(q^{2} ; D\right)+\left(\Delta^{2}(D) / q^{2}\right)},
$$

which is not surprising, since the original gluon SD equation (2.12) and its modified version (2.15) differ from each other only by the longitudinal (unphysical) part.

However, the important observation is that now it is not required to put the mass gap $\Delta^{2}(D)$ formally zero everywhere. The spurious mechanism does not affect the dynamical context of the original gluon SD equation. In other words, it makes it possible to retain the mass gap in the transversal part of the gluon SD equation, and, at the same time, to cancel the term in its longitudinal part, which violates the ST identity. In this way, the modified gluon SD equation (2.15) satisfies automatically the ST identity (2.10).

Due to AF in QCD the PT regime is realized at $q^{2} \rightarrow \infty$. In this limit all the Green's functions are possible to approximate by their free PT counterparts (up to the corresponding PT logarithms). However, from the relation (2.16) it follows that in this limit the mass gap term contribution $\Delta^{2}(D) / q^{2}$ is only next-to-next-to-leading order one. The leading order contribution is the subtracted gluon self-energy $\Pi^{s}\left(q^{2} ; D\right)$, which behaves like $\ln q^{2}$ in this limit, as mentioned above. The constant 1 is the next-to-leading order term in the $q^{2} \rightarrow \infty$ limit. Such a special structure of the relation (2.16), namely the mass gap enters it through the combination $\Delta^{2}(D) / q^{2}$ in its denominator only, explains immediately why the mass gap $\Delta^{2}(D)$ is not important in PT. From this structure it follows that the PT regime at $q^{2} \rightarrow \infty$ is effectively equivalent to the formal $\Delta^{2}(D)=0$ limit and vice versa. That is the reason why this limit can be called the PT limit. And that is why we denote $D_{\mu \nu}\left(q ; \Delta^{2}=0\right)=D_{\mu \nu}(q ; 0) \equiv D_{\mu \nu}^{P T}(q)$, and hence $d\left(q^{2} ; \Delta^{2}=0\right)=d\left(q^{2} ; 0\right) \equiv d^{P T}\left(q^{2}\right)$, etc., in accordance with the previous notations. Let us note, however, that sometimes it is useful to distinguish between the asymptotic suppression of the mass gap contribution $\Delta^{2} / q^{2}$ in the $q^{2} \rightarrow \infty$ limit and the formal PT $\Delta^{2}=0$ limit (see our subsequent paper).

Thus the formal PT $\Delta^{2}(D)=0$ limit exists, and it is a regular one. As it follows from above, in this limit one recovers the PT QCD system of equations (2.13)-(2.14) from the NP QCD one (2.15)-(2.16). So, we distinguish between the PT and NP phases in QCD by the explicit presence of the mass gap. Its aim is to be responsible for the NP QCD dynamics, since it dominates at $q^{2} \rightarrow 0$ in the "solution" (2.16). When it is put formally zero, then the PT phase survives only. Evidently, when such a scale is explicitly present then the QCD coupling constant plays no role in the NP QCD dynamics.

\section{E. Transversality of the relevant full gluon propagator}

The NP QCD system of equations(2.15)-(2.16) depends explicitly on the mass gap $\Delta^{2}$. As it has been discussed in detail in our previous work [4], then the ghosts are not able to cancel the longitudinal component in the full gluon propagator, i.e., they are of no use in this case (the transversality condition for the full gluon self-energy is always violated, see relations (2.8) and (2.9)). This is the price we have paid to keep the mass gap "alive" in the full gluon propagator. Our aim here is to formulate a method which allows one to make the gluon propagator, relevant for NP QCD, purely transversal in a gauge invariant way, even if the mass gap is explicitly present.

For this purpose let us define the truly NP (TNP) part of the full gluon propagator as follows:

$$
D_{\mu \nu}^{T N P}\left(q ; \Delta^{2}\right)=D_{\mu \nu}\left(q ; \Delta^{2}\right)-D_{\mu \nu}\left(q ; \Delta^{2}=0\right)=D_{\mu \nu}\left(q ; \Delta^{2}\right)-D_{\mu \nu}^{P T}(q),
$$

i.e., the subtraction is made with respect to the mass gap $\Delta^{2}$, and therefore the separation between these two terms is exact. So it becomes 


$$
D_{\mu \nu}^{T N P}\left(q ; \Delta^{2}\right)=i T_{\mu \nu}(q)\left[d\left(q^{2} ; \Delta^{2}\right)-d^{P T}\left(q^{2}\right)\right] \frac{1}{q^{2}}=i T_{\mu \nu}(q) d^{T N P}\left(q^{2} ; \Delta^{2}\right) \frac{1}{q^{2}},
$$

where the explicit expression for the TNP Lorentz structure $d^{T N P}\left(q^{2} ; \Delta^{2}\right)=d\left(q^{2} ; \Delta^{2}\right)-d^{P T}\left(q^{2}\right)$ can be obtained from the relations $(2.16)$ and $(2.14)$ for $d\left(q^{2} ; \Delta^{2}\right)$ and $d^{P T}\left(q^{2}\right)$, respectively.

The subtraction (2.17) is equivalent to

$$
D_{\mu \nu}\left(q ; \Delta^{2}\right)=D_{\mu \nu}^{T N P}\left(q ; \Delta^{2}\right)+D_{\mu \nu}^{P T}(q) .
$$

The TNP gluon propagator (2.18) does not survive in the formal PT $\Delta^{2}=0$ limit. This means that it is free of the PT contributions, by construction. The full gluon propagator in this limit is reduced to its PT counterpart. This means that the full gluon propagator, being also NP, nevertheless, is "contaminated" by them. The TNP gluon propagator is purely transversal in a gauge invariant way (no special (Landau) gauge choice by hand), while its full counterpart has a longitudinal component as well. There is no doubt that the true NP dynamics of the full gluon propagator is completely contained in its TNP part, since the subtraction (2.19) is nothing but adding zero to the full gluon propagator. We can write $D_{\mu \nu}\left(q ; \Delta^{2}\right)=i\left\{T_{\mu \nu}(q) d\left(q^{2} ; \Delta^{2}\right)+\xi L_{\mu \nu}(q)\right\}\left(1 / q^{2}\right)-i T_{\mu \nu}(q) d^{P T}\left(q^{2}\right)\left(1 / q^{2}\right)+i T_{\mu \nu}(q) d^{P T}\left(q^{2}\right)\left(1 / q^{2}\right)=$ $D_{\mu \nu}^{T N P}\left(q ; \Delta^{2}\right)+D_{\mu \nu}^{P T}(q)$, and so the true NP dynamics in the full gluon propagator is not affected, but contrary exactly separated from its PT dynamics, indeed. In other words, the TNP gluon propagator is the full gluon propagator but free of its PT "tail".

Taking this important observation into account, we propose instead of the full gluon propagator to use its TNP counterpart (2.18) as the relevant gluon propagator for NP QCD, i.e., to replace

$$
D_{\mu \nu}\left(q ; \Delta^{2}\right) \rightarrow D_{\mu \nu}^{T N P}\left(q ; \Delta^{2}\right)=D_{\mu \nu}\left(q ; \Delta^{2}\right)-D_{\mu \nu}^{P T}(q),
$$

and hence $d\left(q^{2} ; \Delta^{2}\right) \rightarrow d^{T N P}\left(q^{2} ; \Delta^{2}\right)=d\left(q^{2} ; \Delta^{2}\right)-d^{P T}\left(q^{2}\right)$.

The subtraction (2.20) plays effectively the role of ghosts in our proposal. However, the ghosts cancel only the longitudinal component in the PT gluon propagator, while our proposal leads to the cancellation of the PT contribution in the full gluon propagator as well (and thus to an automatical cancellation of its longitudinal component). Nevertheless, this is not a problem, since the mass gap is not survived in the formal PT limit, anyway.

In fact, our proposal is reduced to a rather simple prescription. If one knows a full gluon propagator, and is able to identify the mass scale parameter responsible for the NP dynamics in it, then the full gluon propagator should be replaced in accordance with the subtraction (2.20). The only problem with it is that, being exact, it may not be unique. However, the uniqueness of such kind of separation can be achieved only in the explicit solution for the full gluon propagator as a function of the mass gap (see below). Anyway, this subtraction is a first necessary step, which guarantees transversality of the TNP gluon propagator $D_{\mu \nu}^{T N P}\left(q ; \Delta^{2}\right)$ without losing even one bit of information on the true NP dynamics in the full gluon propagator $D_{\mu \nu}\left(q ; \Delta^{2}\right)$. At the same time, its non-trivial PT dynamics is completely saved in its PT part $D_{\mu \nu}^{P T}(q)$. So it is worth emphasizing that the both terms in the subtraction (2.19) are valid in the whole momentum range, i.e., they are not asymptotics.

The full gluon propagator (2.19), keeping the mass gap "alive", is not "physical" in the sense that it cannot be made transversal by ghosts. Therefore it cannot be used for numerical calculations of the physical observables from first principles. However, our proposal makes it possible to present it as the exact sum of the two "physical" propagators. The TNP gluon propagator is automatically transversal, by construction. It fully contains all the information of the full gluon propagator on its NP context. Just it should be used in accordance with the prescription (2.20) in order to calculate the physical observables in low-energy QCD. In high-energy QCD the PT gluon propagator (2.13) is to be used. It is free of the mass gap and the ghosts can cancel its longitudinal component, making it thus transversal ("physical").

Concluding, in this section we have briefly remind how to preserve the color gauge invariance/symmetry in QCD at non-zero mass gap. This means that from now on we can forget the relations (2.8) and (2.9) at all, since there are no any more their negative consequences for the truly NP QCD. In this connection let us remind the initial subtraction (2.3) has been done in a gauge invariant way (i.e., not in a separate propagators, which enter the skeleton loop integrals, contributing to the full gluon self-energy).

\section{MASSIVE SOLUTION}

One of the direct consequences of the explicit presence of the mass gap in the full gluon propagator is that the gluon may acquire an effective mass, indeed [5]. From Eq. (2.16) it follows that 


$$
\frac{1}{q^{2}} d\left(q^{2}\right)=\frac{1}{q^{2}+q^{2} \Pi^{s}\left(q^{2} ; \xi\right)+\Delta^{2} c(\xi)},
$$

where instead of the dependence on $D$ the dependence on $\xi$ is explicitly shown, while here and below the dependence on all other parameters is not shown, for simplicity. The full gluon propagator (2.11) may have a pole-type solution at the finite point if and only if the denominator in Eq. (3.1) has a zero at this point $q^{2}=-m_{g}^{2}$ (Euclidean signature), i.e.,

$$
-m_{g}^{2}-m_{g}^{2} \Pi^{s}\left(-m_{g}^{2} ; \xi\right)+\Delta^{2} c(\xi)=0
$$

where $m_{g}^{2} \equiv m_{g}^{2}(\xi)$ is an effective gluon mass. The previous equation is a transcendental equation for its determination. Evidently, the number of its solutions is not fixed, a priori. Excluding the mass gap, one obtains that the denominator in the full gluon propagator becomes

$$
q^{2}+q^{2} \Pi^{s}\left(q^{2} ; \xi\right)+\Delta^{2} c(\xi)=q^{2}+m_{g}^{2}+q^{2} \Pi^{s}\left(q^{2} ; \xi\right)+m_{g}^{2} \Pi^{s}\left(-m_{g}^{2} ; \xi\right) .
$$

Let us now expand $\Pi^{s}\left(q^{2} ; \xi\right)$ in a Taylor series near $m_{g}^{2}$ :

$$
\Pi^{s}\left(q^{2} ; \xi\right)=\Pi^{s}\left(-m_{g}^{2} ; \xi\right)+\left(q^{2}+m_{g}^{2}\right) \Pi^{\prime s}\left(-m_{g}^{2} ; \xi\right)+O\left(\left(q^{2}+m_{g}^{2}\right)^{2}\right)
$$

Substituting this expansion into the previous relation and after doing some tedious algebra, one obtains

$$
q^{2}+m_{g}^{2}+q^{2} \Pi^{s}\left(q^{2} ; \xi\right)+m_{g}^{2} \Pi^{s}\left(-m_{g}^{2} ; \xi\right)=\left(q^{2}+m_{g}^{2}\right)\left[1+\Pi^{s}\left(-m_{g}^{2} ; \xi\right)-m_{g}^{2} \Pi^{\prime s}\left(-m_{g}^{2} ; \xi\right)\right]\left[1+\Pi^{s, R}\left(q^{2} ; \xi\right)\right]
$$

where $\Pi^{s, R}\left(q^{2} ; \xi\right)=0$ at $q^{2}=-m_{g}^{2}$ and it is regular at small $q^{2}$; otherwise it remains arbitrary.

The full gluon propagator (2.11) thus now looks

$$
D_{\mu \nu}\left(q ; m_{g}^{2}\right)=i T_{\mu \nu}(q) \frac{Z_{3}\left(m_{g}^{2}\right)}{\left(q^{2}+m_{g}^{2}\right)\left[1+\Pi^{s, R}\left(q^{2} ; m_{g}^{2}\right)\right]}+i \xi L_{\mu \nu}(q) \frac{1}{q^{2}}
$$

where, for future purpose, in the invariant function $\Pi^{s, R}\left(q^{2} ; m_{g}^{2}\right)$ instead of the gauge-fixing parameter $\xi$ we introduced the dependence on the gluon effective mass squared $m_{g}^{2}$, which depends on $\xi$ itself. The gluon propagator's renormalization constant is

$$
Z_{3}\left(m_{g}^{2}\right)=\frac{1}{1+\Pi^{s}\left(-m_{g}^{2} ; \xi\right)-m_{g}^{2} \Pi^{\prime s}\left(-m_{g}^{2} ; \xi\right)}
$$

In the formal PT limit $\Delta^{2}=0$, an effective gluon mass is also zero, $m_{g}^{2}(\xi)=0$, as it follows from Eq. (3.2). So an effective gluon mass is the NP effect. At the same time, it cannot be interpreted as the "physical" gluon mass, since it remains explicitly gauge-dependent quantity (at least at this stage). In other words, we were unable to renormalize it along with the gluon propagator (3.6). In the formal PT $\Delta^{2}=m_{g}^{2}(\xi)=0$ limit the gluon propagator's renormalization constant (3.7) becomes the standard one [1, 2], namely

$$
Z_{3}(0)=\frac{1}{1+\Pi^{s}(0 ; \xi)}
$$

It is interesting to note that Eq. (3.2) has a second solution in the formal PT $\Delta^{2}=0$ limit. In this case an effective gluon mass remains finite, but $1+\Pi^{s}\left(-m_{g}^{2} ; \xi\right)=0$. So a scale responsible for the NP dynamics is not determined by an effective gluon mass itself, but by this condition. Its interpretation from the physical point of view is not clear. The massive solution (3.6) is difficult to use for the solution of the color confinement problem, since it is smooth in the $q^{2} \rightarrow 0$ limit. However, its existence shows the general possibility for a vector particles to acquire masses dynamically, i.e., without so-called Higgs mechanism [6], which requires the existence of not yet discovered Higgs 
particle. Apparently, it can be also useful in the generalization of QCD to non-zero temperature and density [7, 8] (and references therein), when the gluons may indeed acquire effective masses. The above-mentioned possibility is due only to the internal dynamics and symmetries of the corresponding gauge theory.

The general procedure described above in subsection E of section II can be directly applied to the massive solution (3.6). So it becomes

$$
D_{\mu \nu}\left(q ; m_{g}^{2}\right)=D_{\mu \nu}^{T N P}\left(q ; m_{g}^{2}\right)+D_{\mu \nu}^{P T}(q)
$$

where

$$
D_{\mu \nu}^{T N P}\left(q ; m_{g}^{2}\right)=i T_{\mu \nu}(q)\left[\frac{Z_{3}\left(m_{g}^{2}\right)}{\left(q^{2}+m_{g}^{2}\right)\left[1+\Pi^{s, R}\left(q^{2} ; m_{g}^{2}\right)\right]}-\frac{Z_{3}(0)}{q^{2}\left[1+\Pi^{s, R}\left(q^{2} ; 0\right)\right]}\right]
$$

and

$$
D_{\mu \nu}^{P T}(q)=i\left[T_{\mu \nu}(q) \frac{Z_{3}(0)}{\left[1+\Pi^{s, R}\left(q^{2} ; 0\right)\right]}+\xi L_{\mu \nu}(q)\right] \frac{1}{q^{2}} .
$$

Let us remind that in the massive solution the role of the mass gap is played by an effective gluon mass, so the formal PT limit is $m_{g}^{2}=0$. In accordance with our prescription (2.20), we should finally replace the full gluon propagator (3.6) as follows: $D_{\mu \nu}\left(q ; m_{g}^{2}\right) \rightarrow D_{\mu \nu}^{T N P}\left(q ; m_{g}^{2}\right)$, where the latter is explicitly given in Eq. (3.10).

\section{GENERAL NL ITERATION SOLUTION}

In order to find another type of the general formal solution for the full gluon propagator (2.15), let us begin again with its "solution" (2.16) which is

$$
d\left(q^{2}\right) \equiv d\left(q^{2} ; \Delta^{2}\right)=\frac{1}{1+\Pi^{s}\left(q^{2} ; d\right)+c(d)\left(\Delta^{2} / q^{2}\right)}
$$

where the dependence on $D$ is replaced by the equivalent dependence on $d$ and the relation (2.4) is already used. It is worth reminding that the invariant function $\Pi^{s}\left(q^{2} ; d\right)$ and $c(d)$ are, in fact, the sum of the corresponding skeleton loop integrals (see section II and our initial paper [4] ). Let us introduce further the dimensionless variable $z=\Delta^{2} / q^{2}$. The full Lorentz structure (4.1) regularly depends on the mass gap, and hence on $z$. Thus it can be expand in a Taylor series in powers of $z$ around zero $z$ as follows:

$$
d\left(q^{2} ; \Delta^{2}\right)=d\left(q^{2} ; z\right)=\sum_{k=0}^{\infty} z^{k} f_{k}\left(q^{2}\right)
$$

where the functions $f_{k}\left(q^{2}\right)$ are the corresponding derivatives of $d\left(q^{2} ; z\right)$ with respect to $z$ at $z=0$, which is equivalent to the PT $\Delta^{2}=0$ limit. For example, $f_{0}\left(q^{2}\right)=d\left(q^{2} ; z=0\right)=d^{P T}\left(q^{2}\right)=\left[1+\Pi^{s}\left(q^{2} ; d^{P T}\right]^{-1}, f_{1}\left(q^{2}\right)=\right.$ $\left(\partial d\left(q^{2} ; z\right) / \partial z\right)_{z=0}=\left[\partial\left[1+\Pi^{s}\left(q^{2} ; d\right)+c(d) z\right]^{-1} / \partial z\right]_{z=0}=-\left[1+\Pi^{s}\left(q^{2} ; d^{P T}\right)\right]^{-2} c\left(d^{P T}\right)=-\left[d^{P T}\left(q^{2}\right)\right]^{2} c\left(d^{P T}\right)$, and so on, i.e., $f_{k}\left(q^{2}\right)=(-1)^{k} d^{P T}\left(q^{2}\right)\left[d^{P T}\left(q^{2}\right) c\left(d^{P T}\right)\right]^{k}$. Fortunately, these explicit expressions play no any role in what follows. In any case, they depend on the unknown, in general, quantities $\Pi^{s}\left(q^{2} ; d\right)$ and $c(d)$, which by themselves NL depend on $d$ and finally on $d^{P T}$ and $c\left(d^{P T}\right)$. So our expansion (4.2) is nothing but the NL iteration series in powers of the mass gap (for the direct NL iteration procedure with $d^{(0)}=1$ as input information see appendix A). To use also unknown functions $f_{k}\left(q^{2}\right)$ much more convenient from the technical point of view. However, it is worth emphasizing that, contrary to the relation (4.1), the expansion (4.2) can be considered now as a formal solution for $d\left(q^{2}\right)$, since $f_{k}\left(q^{2}\right)$ depend on $d^{P T}\left(q^{2}\right)$, which is assumed to be "known".

The functions $f_{k}\left(q^{2}\right)$ are regular functions of the variable $q^{2}$, since they finally depend on $d^{P T}\left(q^{2}\right)$ which is a regular function of $q^{2}$. Therefore they can be expand in a Taylor series near $q^{2}=0$ (here we can put the subtraction point $\alpha=0$, for simplicity, since all the quantities are already regularized, i.e., they depend on $\alpha$ and so on, see appendix A). 
Introducing the dimensionless variable $x=q^{2} / M^{2}$, where $M^{2}$ is some fixed auxiliary mass squared, it is convenient to present this expansion as a sum of the two terms, namely

$$
f_{k}\left(q^{2}\right)=\sum_{n=0}^{k} x^{n} f_{k n}(0)+x^{k+1} B_{k}(x)
$$

where the coefficient $f_{k n}(0)$ are the corresponding derivatives of the functions $f_{k}\left(q^{2}\right) \equiv f_{k}(x)$ with respect to $x$ at $x=0$. Of course, these coefficients depend on the parameters of the theory such as $\lambda, \alpha, \xi, g^{2}$, and so on, which are not shown explicitly. The dependence on these parameters will be restored at the final stage of our derivations. The dimensionless functions $B_{k}(x)$ are regular functions of $x$; otherwise they remain arbitrary.

So the general Lorentz structure (4.2) becomes

$$
d\left(q^{2}\right)=\sum_{k=0}^{\infty} z^{k} f_{k}(x)=\sum_{k=0}^{\infty} z^{k}\left(\sum_{n=0}^{k} x^{n} f_{k n}(0)+x^{k+1} B_{k}(x)\right) .
$$

Omitting all the intermediate tedious derivations (which, nevertheless, are quite obvious), these double sums can be equivalently present as the sum of the three independent terms as follows:

$$
d\left(q^{2}\right)=z \sum_{k=0}^{\infty} z^{k} \sum_{m=0}^{\infty} \Phi_{k m}(0)+a \sum_{k=0}^{\infty} a^{k} \sum_{m=0}^{\infty} A_{k m}(x)+d^{P T}\left(q^{2}\right),
$$

where the constant $a=x z=\Delta^{2} / M^{2}$ and the dimensionless functions $A_{k m}(x)$ are regular functions of $x$ : otherwise they remain arbitrary. $d^{P T}\left(q^{2}\right)$ denotes the terms which do not depend on the mass gap $\Delta^{2}$ at all, i.e., it is nothing but the Lorentz structure of the PT gluon propagator (2.14), indeed. The summation over $m$ explicitly shows that all iterations invoke each NP IR singularity labeled by $k$ in the first term of the expansion (4.5). Thus it is the general NL formal expansion in powers of the mass gap (this is explicitly seen from appendix A).

Going back to the gluon momentum variable $q^{2}$, one obtains

$$
d\left(q^{2} ; \Delta^{2}\right)=d^{T N P}\left(q^{2} ; \Delta^{2}\right)+d^{P T}\left(q^{2}\right)=d^{I N P}\left(q^{2} ; \Delta^{2}\right)+d^{M P T}\left(q^{2} ; \Delta^{2}\right)+d^{P T}\left(q^{2}\right),
$$

where the superscripts "INP" and "MPT" stand for the intrinsically NP and mixed PT parts of the TNP term, respectively (for reasons see discussion below). In other words, in the general NL iteration solution the TNP part itself is a sum of the two independent terms, i.e., $d^{T N P}\left(q^{2} ; \Delta^{2}\right)=d^{I N P}\left(q^{2} ; \Delta^{2}\right)+d^{M P T}\left(q^{2} ; \Delta^{2}\right)$. Their explicit expressions are

$$
d^{I N P}\left(q^{2} ; \Delta^{2}\right)=\left(\frac{\Delta^{2}}{q^{2}}\right) \sum_{k=0}^{\infty}\left(\frac{\Delta^{2}}{q^{2}}\right)^{k} \Phi_{k}=\left(\frac{\Delta^{2}}{q^{2}}\right) \sum_{k=0}^{\infty}\left(\frac{\Delta^{2}}{q^{2}}\right)^{k} \sum_{m=0}^{\infty} \Phi_{k m}
$$

and

$$
d^{M P T}\left(q^{2} ; \Delta^{2}\right)=\left(\frac{\Delta^{2}}{M^{2}}\right) \sum_{k=0}^{\infty}\left(\frac{\Delta^{2}}{M^{2}}\right)^{k} A_{k}\left(q^{2}\right)=\left(\frac{\Delta^{2}}{M^{2}}\right) \sum_{k=0}^{\infty}\left(\frac{\Delta^{2}}{M^{2}}\right)^{k} \sum_{m=0}^{\infty} A_{k m}\left(q^{2}\right) .
$$

Here and everywhere below all the quantities depend on the parameters of the theory, namely $\Delta^{2}=\Delta^{2}\left(\lambda, \alpha, \xi, g^{2}\right)$ and $A_{k}\left(q^{2}\right)=\sum_{m=0}^{\infty} A_{k m}\left(q^{2} ; \lambda, \alpha, \xi, g^{2}\right)$. At the same time, $\Phi_{k m}$ depends in addition on the parameter $a$ as well, i.e., $\Phi_{k m}=\Phi_{k m}\left(\lambda, \alpha, \xi, g^{2}, a\right)$.

\section{A. The exact structure of the NL iteration solution}

The full gluon propagator (2.11) thus becomes the sum of the three independent terms, namely 


$$
D_{\mu \nu}\left(q ; \Delta^{2}\right)=D_{\mu \nu}^{T N P}\left(q ; \Delta^{2}\right)+D_{\mu \nu}^{P T}(q)=D_{\mu \nu}^{I N P}\left(q ; \Delta^{2}\right)+D_{\mu \nu}^{M P T}\left(q ; \Delta^{2}\right)+D_{\mu \nu}^{P T}(q),
$$

where

$$
D_{\mu \nu}^{I N P}\left(q ; \Delta^{2}\right)=i T_{\mu \nu}(q) d^{I N P}\left(q^{2} ; \Delta^{2}\right) \frac{1}{q^{2}}=i T_{\mu \nu}(q) \frac{\Delta^{2}}{\left(q^{2}\right)^{2}} L\left(q^{2} ; \Delta^{2}\right)
$$

with

$$
L\left(q^{2} ; \Delta^{2}\right)=\sum_{k=0}^{\infty}\left(\frac{\Delta^{2}}{q^{2}}\right)^{k} \Phi_{k}=\sum_{k=0}^{\infty}\left(\frac{\Delta^{2}}{q^{2}}\right)^{k} \sum_{m=0}^{\infty} \Phi_{k m}
$$

while

$$
D_{\mu \nu}^{M P T}\left(q ; \Delta^{2}\right)=i T_{\mu \nu}(q) d^{M P T}\left(q^{2} ; \Delta^{2}\right) \frac{1}{q^{2}}
$$

with $d^{M P T}\left(q^{2} ; \Delta^{2}\right)$ given in Eq. (4.13) and

$$
D_{\mu \nu}^{P T}(q)=i\left[T_{\mu \nu}(q) d^{P T}\left(q^{2}\right)+\xi L_{\mu \nu}(q)\right] \frac{1}{q^{2}}
$$

with $d^{P T}\left(q^{2}\right)$ given in Eq. (2.14). For the direct NL iteration procedure see appendix A, as mentioned above.

Let us emphasize that the general problem of convergence of formal (but regularized) series, which appear in these relations, is irrelevant here. In other words, it does not make any sense to discuss the convergence of such kind of series before the renormalization program is performed (which will allow one to see whether or not the mass gap survives it at all). The problem how to remove the UV overlapping divergences [9] and usual overall ones [1, 2, 10, 11] is a standard one, i.e., it is not our problem, anyway (let us remind that the mass gap does not survive in the PT $q^{2} \rightarrow \infty$ limit). Our problem will be how to deal with severe infrared (IR) $\left(q^{2} \rightarrow 0\right)$ singularities due to their novelty and genuine (intrinsic) NP character (in this limit the mass gap dominates the structure of the full gluon propagator). Fortunately, there already exists a well-elaborated mathematical formalism for this purpose, namely the distribution theory (DT) 12], into which the dimensional regularization method (DRM) [13] should be correctly implemented (see also Refs. [14, 15]).

The INP part of the full gluon propagator is characterized by the presence of severe power-type (or, equivalently, NP) IR singularities $\left(q^{2}\right)^{-2-k}, k=0,1,2,3, \ldots$ So these IR singularities are defined as more singular than the powertype IR singularity of the free gluon propagator $\left(q^{2}\right)^{-1}$, which thus can be defined as the PT IR singularity. The INP part of the full gluon propagator (4.10), apart from the structure $\left(\Delta^{2} / q^{4}\right)$, is nothing but the corresponding Laurent expansion (explicitly shown in Eq. (4.11)) in integer powers of $q^{2}$ accompanied by the corresponding powers of the mass gap squared and multiplied by the $q^{2}$-independent factors, the so-called residues $\Phi_{k}\left(\lambda, \alpha, \xi, g^{2}, a\right)=$ $\sum_{m=0}^{\infty} \Phi_{k m}\left(\lambda, \alpha, \xi, g^{2}, a\right)$. The sum over $m$ indicates that an infinite number of iterations (all iterations) of the abovementioned corresponding regularized skeleton loop integrals invokes each severe IR singularity labeled by $k$. It is worth emphasizing that the Laurent expansion (4.11) cannot be summed up into the some known function, since its residues are, in general, arbitrary. However, this arbitrariness is not a problem. The functional dependence, which has been established exactly, is all that matters (this will be explicitly shown in the subsequent paper). Let us note that the expansions (4.10)-(4.11) have been independently obtained in Ref. [14] in a rather different way.

The MPT part of the full gluon propagator (4.12), which has the power-type PT IR singularity only, remains undetermined, but depends on the mass gap (that is why we call this term as the mixed PT contribution, but it vanishes in the formal PT $\Delta^{2}=0$ limit). This is the price we have paid to fix exactly the functional dependence of the INP part of the full gluon propagator. With respect to the character of the IR singularity it should be combined with the PT gluon propagator, leading to the so-called general PT (GPT) term, namely

$$
D_{\mu \nu}^{G P T}\left(q ; \Delta^{2}\right)=D_{\mu \nu}^{M P T}\left(q ; \Delta^{2}\right)+D_{\mu \nu}^{P T}(q)=i\left[T_{\mu \nu}(q) d^{G P T}\left(q^{2} ; \Delta^{2}\right)+\xi L_{\mu \nu}(q)\right] \frac{1}{q^{2}},
$$


where $d^{G P T}\left(q^{2} ; \Delta^{2}\right)=d^{M P T}\left(q^{2} ; \Delta^{2}\right)+d^{P T}\left(q^{2}\right)$ is regular at small $q^{2}$, while $d^{M P T}\left(q^{2} ; \Delta^{2}=0\right)=0$ and hence $d^{G P T}\left(q^{2} ; \Delta^{2}=0\right)=d^{P T}\left(q^{2}\right)$. Thus both terms MPT and PT present the PT-type contributions to the full gluon propagator (4.6). It is worth reminding that all the three terms, which appear in the right-hand-side of Eq. (4.9) are valid in the whole energy/momentum range, i.e., they are not asymptotics. At the same time, we have achieved the separation between the terms responsible for the NP (dominating in the $\mathrm{IR}\left(q^{2} \rightarrow 0\right)$ ) and the nontrivial PT (dominating in the UV $\left(q^{2} \rightarrow \infty\right)$ ) dynamics in the true QCD vacuum. The structure of this solution shows clearly that the deep IR region interesting for confinement and other NP effects is dominated by the mass gap. In the formal $\mathrm{PT} \Delta^{2}=0$ limit, the nontrivial PT dynamics is all that matters.

\section{INP GLUON PROPAGATOR}

In accordance with our prescription, one should subtract all the types of the PT contributions in order to get the relevant gluon propagator for the truly NP QCD. As it follows from discussion above, in the case of the NL iteration solution, we should subtract the two terms. Doing so in Eq. (4.9), on account of Eq. (4.14), one finally obtains

$$
D_{\mu \nu}\left(q ; \Delta^{2}\right) \rightarrow D_{\mu \nu}^{I N P}\left(q ; \Delta^{2}\right)=D_{\mu \nu}\left(q ; \Delta^{2}\right)-D_{\mu \nu}^{G P T}\left(q ; \Delta^{2}\right),
$$

and hence $d\left(q^{2}\right) \rightarrow d^{I N P}\left(q^{2}\right)$ as well, so that

$$
D_{\mu \nu}^{I N P}\left(q ; \Delta^{2}\right)=i T_{\mu \nu}(q) \frac{\Delta^{2}}{\left(q^{2}\right)^{2}} L\left(q^{2} ; \Delta^{2}\right)=i T_{\mu \nu}(q) \frac{\Delta^{2}}{\left(q^{2}\right)^{2}} \sum_{k=0}^{\infty}\left(\frac{\Delta^{2}}{q^{2}}\right)^{k} \Phi_{k}
$$

where $\Delta^{2}=\Delta^{2}\left(\lambda, \alpha, \xi, g^{2}\right)$ and $\Phi_{k}=\Phi_{k}\left(\lambda, \alpha, \xi, g^{2}\right)=\sum_{m=0}^{\infty} \Phi_{k m}\left(\lambda, \alpha, \xi, g^{2}\right)$. In this connection, let us note that after the subtraction (5.1) is completed we can put the intermediate parameter $a=1$, to equate thus the auxiliary fixed mass to the mass gap itself, i.e., put $M^{2}=\Delta^{2}$, not losing generality. In the deep IR region $\left(q^{2} \rightarrow 0\right)$ the mass gap is only one that's really matters. All other masses introduced from a technical point of view in order to clarify the derivations play only auxiliary role.

It is important to emphasize that the INP gluon propagator (5.2) is uniquely defined because there exists a special regularization expansion for severe (i.e., NP) IR singularities, while for the PT IR singularity such kind of expansion does not exist at all (see Refs. [12, 14, 15] and references therein). This just determines the principal difference between the NP and PT IR singularities. It is also exactly defined because of its two features. The first one is that the INP gluon propagator depends only on the transversal degrees of freedom of gauge bosons. The second one is that in the formal PT $\Delta^{2}=0$ limit the INP gluon propagator vanishes. Thus, one can conclude that the presence of severe IR singularities only is the first necessary condition, while the regular dependence on the mass gap and transversality is only second sufficient condition for the unique and exact separation of the INP gluon propagator from the PT gluon propagator. At the same time, the TNP gluon propagator is not uniquely defined, since it contains the MPT part, see Eq. (4.9). In other words, the INP gluon propagator is free of all the types of the PT contributions ("contaminations"). Just it should replace the full gluon propagator in order to calculate the physical observables, processes, etc. from first principles in low-energy QCD after the corresponding renormalization program is performed.

The INP gluon propagator satisfies its own equation of motion. For the sake of completeness, let us begin with the SD equation for the TNP gluon propagator [4], namely

$$
\begin{aligned}
D_{\mu \nu}^{T N P}\left(q ; \Delta^{2}\right) & =D_{\mu \rho}^{0}(q) i T_{\rho \sigma}(q)\left[-q^{2} \Pi^{s}\left(q^{2} ; D^{P T}\right)+q^{2} \Pi^{s}\left(q^{2} ; D\right)+\Delta^{2}\right] D_{\sigma \nu}^{P T}(q) \\
& +D_{\mu \rho}^{0}(q) i T_{\rho \sigma}(q)\left[q^{2} \Pi^{s}\left(q^{2} ; D\right)+\Delta^{2}\right] D_{\sigma \nu}^{T N P}\left(q ; \Delta^{2}\right)
\end{aligned}
$$

with

$$
D_{\mu \nu}^{T N P}(q)=i\left\{T_{\mu \nu}(q) d^{T N P}\left(q^{2}\right)+\xi L_{\mu \nu}(q)\right\} \frac{1}{q^{2}}
$$

Here and below we omit the dependence on the mass gap in the propagators and their Lorentz structures, for simplicity. On account of this decomposition, the "solution" of the previous equation is

$$
d^{T N P}\left(q^{2}\right)=\frac{\Pi^{s}\left(q^{2} ; D^{P T}\right)-\Pi^{s}\left(q^{2} ; D\right)-\left(\Delta^{2} / q^{2}\right)}{\left[1+\Pi^{s}\left(q^{2} ; D\right)+\left(\Delta^{2} / q^{2}\right)\right]\left[1+\Pi^{s}\left(q^{2} ; D^{P T}\right)\right]}
$$


This expression coincides with the definition of $d^{T N P}\left(q^{2}\right)=d\left(q^{2}\right)-d^{P T}\left(q^{2}\right)$ on account of the explicit expressions (2.14) and (2.15), as it should be.

From Eq. (4.9) it follows that

$$
D_{\mu \nu}^{T N P}(q)=D_{\mu \nu}^{I N P}(q)+D_{\mu \nu}^{M P T}(q),
$$

and substituting it into Eq. (5.3), one obtains the SD equation for the INP gluon propagator, namely

$$
\begin{aligned}
D_{\mu \nu}^{I N P}(q)= & -D_{\mu \nu}^{M P T}(q)+D_{\mu \rho}^{0}(q) i T_{\rho \sigma}(q)\left[q^{2} \Pi^{s}\left(q^{2} ; D\right)+\Delta^{2}\right] D_{\sigma \nu}^{M P T}(q) \\
& +D_{\mu \rho}^{0}(q) i T_{\rho \sigma}(q)\left[-q^{2} \Pi^{s}\left(q^{2} ; D^{P T}\right)+q^{2} \Pi^{s}\left(q^{2} ; D\right)+\Delta^{2}\right] D_{\sigma \nu}^{P T}(q) \\
& +D_{\mu \rho}^{0}(q) i T_{\rho \sigma}(q)\left[q^{2} \Pi^{s}\left(q^{2} ; D\right)+\Delta^{2}\right] D_{\sigma \nu}^{I N P}(q) .
\end{aligned}
$$

Using the decompositions (2.2), (4.12) and (4.13), it can be simplified to

$$
\begin{aligned}
q^{2} D_{\mu \nu}^{I N P}(q)= & -i T_{\mu \nu}(q)\left(1+\Pi^{s}\left(q^{2} ; D\right)+\left(\Delta^{2} / q^{2}\right)\right) d^{M P T}(q) \\
& -i T_{\mu \nu}(q)\left(-\Pi^{s}\left(q^{2} ; D^{P T}\right)+\Pi^{s}\left(q^{2} ; D\right)+\left(\Delta^{2} / q^{2}\right)\right) d^{P T}(q) \\
& -T_{\mu \sigma}(q) T_{\rho \sigma}(q)\left[q^{2} \Pi^{s}\left(q^{2} ; D\right)+\Delta^{2}\right] D_{\sigma \nu}^{I N P}(q),
\end{aligned}
$$

where $d^{M P T}(q)$ and $d^{P T}(q)$ are given in Eqs. (4.8) and (2.14), respectively. This equation is of no practical use due to its complicated structure. Fortunately, we already have the explicit expression for the INP gluon propagator (4.10)-(4.11) or, equivalently, (5.2). It is only one to be used in order to derive renormalized gluon propagator with the correct confinement properties.

However, from Eq. (5.8) it follows an important observation that like the TNP SD equation (5.3) this equation cannot be reduced to the free gluon propagator, when the interaction is to be switched off (i.e., setting formally $\left.\Pi^{s}\left(q^{2} ; D^{P T}\right)=\Pi^{s}\left(q^{2} ; D\right)=\Delta^{2}=0\right)$. Evidently, to the same conclusion one comes from the explicit expressions (4.8) and (5.5), on account of the relation $d^{I N P}\left(q^{2} ; \Delta^{2}\right)=d^{T N P}\left(q^{2} ; \Delta^{2}\right)-d^{M P T}\left(q^{2} ; \Delta^{2}\right)$, which follows from Eq. (4.6). So in INP QCD the gluon propagator is always "dressed" as well, and thus this theory has no free gluon propagator in its formalism. As it has been argued in our initial work [4], it makes it possible to suppress the emission and absorbtion of the colored dressed gluons at large distances by the renormalization of the mass gap. Both the suppression of the dressed gluons and the absence of the free gluons are necessary for the explanation of gluon confinement by INP QCD (see our next paper). On the other hand, the full gluon propagator (2.19) which satisfies Eq. (2.15) is reduced to the free gluon propagator when the interaction is switched off. There is no mechanism to suppress the emission and absorbtion of the free gluons at large distances [4]. That is why the full gluon propagator (2.19) is not confining, while the INP one (5.2) can be.

The subtraction (5.1) seems to be necessary, indeed. It makes the relevant gluon propagator (5.2) transversal and excludes the free gluons from the theory at the same time.

\section{CONCLUSIONS}

The structure of the full gluon propagator in the presence of the regularized mass gap has been firmly established. We have shown explicitly that in its presence at least two independent and different typed of formal solutions for the regularized full gluon propagator exist. No truncations/approximations/assumptions are made in order to show the existence of these general types of solutions. Also, our approach, in general, and the above-mentioned solutions, in particular, is gauge-invariant, since no special gauge has been chosen. Let us emphasize that before the renormalization program is performed the gauge invariance should be understood in this sense only.

In the presence of the mass gap the gluons may acquire an effective gluon masses, depending on the gauge choice (the so-called massive solution (3.6)), but a gauge-fixing parameter remains arbitrary, i.e., a gauge is not fixed by hand (see remarks above). Its relation to the solution of the color confinement problem is not clear, even after the renormalization program is performed.

The general NL iteration solution (4.9)-(4.13) for the full gluon propagator depends explicitly on the mass gap. It is always severely singular in the $q^{2} \rightarrow 0$ limit, so the gluons remain massless, and this does not depend on the gauge choice. However, we argued that only the INP gluon propagator (5.2) is to be used for the numerical calculations of physical observables, processes, etc. in low-energy QCD from first principles. 
It is worth emphasizing that there exists only one general restriction on the behavior of $\Pi^{s}\left(q^{2} ; D\right)$, which enters the corresponding gluon SD equation (2.15), in the explicit presence of the mass gap within our approach, namely

$$
q^{2} \Pi^{s}\left(q^{2} ; D\right) \rightarrow 0, \quad q^{2} \rightarrow 0,
$$

at any $D$. It stems from the second of the exact decompositions (2.5), since the subtracted gluon self-energy in this limit (or more precisely at $q^{2} \rightarrow \mu^{2}$ ) should go to zero. Otherwise the invariant function $\Pi^{s}\left(q^{2} ; D\right)$ remains arbitrary (but it is logarithmic divergent at infinity). Both general types of formal solutions the massive solution and the NL iteration one satisfy it. The existence of some other solution(s) for the full gluon propagator, satisfying the general condition (6.1), should not be excluded a priori. Let us remind that the gluon SD equation (2.15) is highly NL, so the number of independent solutions is not fixed. Any concrete solution obtained by lattice QCD or by the analytical approach based on the SD system of equations is a particular case of the general types (finite or singular at zero gluon momentum) of the formal solutions established here. They are subject to the different truncations/approximations/assumptions and the concrete gauge choice imposed on the invariant function $\Pi^{s}\left(q^{2} ; D\right)$, which, in general, remains arbitrary but satisfying the above-mentioned general constraint (6.1) within our approach (see, for example recent papers [16, 17, 18, 19, 20, 21, 22] and references therein. Let us also point out Refs. [23, 24, 25, 26] as well, where the gluon propagator is finite and contains the mass scale parameter. However, it, apparently, cannot be interpreted as gluon effective mass).

The INP solution (5.2) is interesting for confinement, but the two important problems remain to solve. The first problem is how to perform the renormalization program for the regularized mass gap $\Delta^{2} \equiv \Delta^{2}\left(\lambda, \alpha, \xi, g^{2}\right)$, and to see whether the mass gap survives it or not (it has been already discussed in our previous work [4]). The second problem is how to treat correctly severe IR singularities $\left(q^{2}\right)^{-2-k}, k=0,1,2,3, \ldots$ inevitably present in this solution (see a few brief remarks above in section IV). Both problems will be addressed and solved in our subsequent paper.

\section{Acknowledgments}

Support by HAS-JINR grant (P. Levai) is to be acknowledged. The author is grateful to P. Forgács, J. Nyiri, C. Wilkin, T. Biró, M. Faber, Á. Lukács, M. Vasúth and especially to A.V. Kouzushin for useful discussions, remarks and help.

\section{APPENDIX A: DIRECT NL ITERATION PROCEDURE}

In order to find a formal solution for the regularized full gluon propagator (2.11), on account of its effective charge (2.16), let us rewrite the latter one in the form of the corresponding transcendental (i.e., not algebraic) equation, namely

$$
d\left(q^{2}\right)=1-\left[\Pi^{s}\left(q^{2} ; d\right)+\frac{\Delta^{2}}{q^{2}} c(d)\right] d\left(q^{2}\right)=1-P\left(q^{2} ; d\right) d\left(q^{2}\right),
$$

where Eq. (2.4) has been already used, and instead of $D$ an equivalent dependence on $d$ is introduced. It is suitable for the formal NL iteration procedure. For future purposes, it is convenient to introduce short-hand notations as follows:

$$
\begin{aligned}
c(d & \left.=d^{(0)}+d^{(1)}+d^{(2)}+\ldots+d^{(m)}+\ldots\right)=c_{m} \equiv c_{m}\left(\lambda, \alpha, \xi, g^{2}\right), \\
\Pi^{s}\left(q^{2} ; d\right. & \left.=d^{(0)}+d^{(1)}+d^{(2)}+\ldots+d^{(m)}+\ldots\right)=\Pi_{m}^{s}\left(q^{2}\right),
\end{aligned}
$$

and

$$
P_{m}\left(q^{2}\right)=\left[\Pi_{m}^{s}\left(q^{2}\right)+\frac{\Delta^{2}}{q^{2}} c_{m}\right], m=0,1,2,3, \ldots
$$

Via the corresponding subscript $m$ it is explicitly seen which iteration for the gluon form factor $d$ is actually done in $c(d), \Pi^{s}\left(q^{2} ; d\right)$ and $P\left(q^{2} ; d\right)$. Let us also point out that all the invariant functions $\Pi_{m}^{s}\left(q^{2}\right)$ can be expand in a formal Taylor series near the finite subtraction point $\alpha$. If it were possible to express the full gluon form factor $d\left(q^{2}\right)$ in 
terms of these quantities then it would be the formal solution for the full gluon propagator. In fact, this is nothing but the skeleton loops expansion, since the regularized skeleton loop integrals, contributing to the gluon self-energy as mentioned above, have to be iterated. This is the so-called general NL iteration solution. This formal expansion is not a PT series. The magnitude of the coupling constant squared and the dependence of the regularized skeleton loop integrals on it is completely arbitrary.

It is instructive to describe the general iteration procedure in some details. Evidently, $d^{(0)}=1$, and this corresponds to the approximation of the full gluon propagator by its free counterpart. Doing the first iteration in Eq. (A1), one thus obtains

$$
d\left(q^{2}\right)=1-P_{0}\left(q^{2}\right)+\ldots=1+d^{(1)}\left(q^{2}\right)+\ldots
$$

where obviously

$$
d^{(1)}\left(q^{2}\right)=-P_{0}\left(q^{2}\right)
$$

Carrying out the second iteration, one gets

$$
d\left(q^{2}\right)=1-P_{1}\left(q^{2}\right)\left[1+d^{(1)}\left(q^{2}\right)\right]+\ldots=1+d^{(1)}\left(q^{2}\right)+d^{(2)}\left(q^{2}\right)+\ldots,
$$

where

$$
d^{(2)}\left(q^{2}\right)=-d^{(1)}\left(q^{2}\right)-P_{1}\left(q^{2}\right)\left[1-P_{0}\left(q^{2}\right)\right] .
$$

Doing the third iteration, one further obtains

$$
d\left(q^{2}\right)=1-P_{2}\left(q^{2}\right)\left[1+d^{(1)}\left(q^{2}\right)+d^{(2)}\left(q^{2}\right)\right]+\ldots=1+d^{(1)}\left(q^{2}\right)+d^{(2)}\left(q^{2}\right)+d^{(3)}\left(q^{2}\right)+\ldots
$$

where

$$
d^{(3)}\left(q^{2}\right)=-d^{(1)}\left(q^{2}\right)-d^{(2)}\left(q^{2}\right)-P_{2}\left(q^{2}\right)\left[1-P_{1}\left(q^{2}\right)\left(1-P_{0}\left(q^{2}\right)\right)\right],
$$

and so on for the next iterations.

Thus up to the third iteration, one finally arrives at

$$
d\left(q^{2}\right)=\sum_{m=0}^{\infty} d^{(m)}\left(q^{2}\right)=1-\left[\Pi_{2}^{s}\left(q^{2}\right)+\frac{\Delta^{2}}{q^{2}} c_{2}\right]\left[1-\left[\Pi_{1}^{s}\left(q^{2}\right)+\frac{\Delta^{2}}{q^{2}} c_{1}\right]\left[1-\Pi_{0}^{s}\left(q^{2}\right)-\frac{\Delta^{2}}{q^{2}} c_{0}\right]\right]+\ldots
$$

We restrict ourselves by the iterated gluon form factor up to the third term, since this already allows to show explicitly some general features of the NL iteration solution.

\section{Splitting/shifting procedure}

Doing some tedious algebra, the previous expression (A10) can be rewritten as follows:

$$
\begin{aligned}
d\left(q^{2}\right) & =\left[1-\Pi_{2}^{s}\left(q^{2}\right)+\Pi_{1}^{s}\left(q^{2}\right) \Pi_{2}^{s}\left(q^{2}\right)-\Pi_{0}^{s}\left(q^{2}\right) \Pi_{1}^{s}\left(q^{2}\right) \Pi_{2}^{s}\left(q^{2}\right)+\ldots\right] \\
& +\frac{\Delta^{2}}{q^{2}}\left[\Pi_{2}^{s}\left(q^{2}\right) c_{1}+\Pi_{1}^{s}\left(q^{2}\right) c_{2}-\Pi_{0}^{s}\left(q^{2}\right) \Pi_{1}^{s}\left(q^{2}\right) c_{2}-\Pi_{0}^{s}\left(q^{2}\right) \Pi_{2}^{s}\left(q^{2}\right) c_{1}-\Pi_{1}^{s}\left(q^{2}\right) \Pi_{2}^{s}\left(q^{2}\right) c_{0}+\ldots\right] \\
& -\frac{\Delta^{4}}{q^{4}}\left[\Pi_{0}^{s}\left(q^{2}\right) c_{1} c_{2}+\Pi_{1}^{s}\left(q^{2}\right) c_{0} c_{2}+\Pi_{2}^{s}\left(q^{2}\right) c_{0} c_{1}+\ldots\right] \\
& -\frac{\Delta^{2}}{q^{2}}\left[c_{2}-\frac{\Delta^{2}}{q^{2}} c_{1} c_{2}+\frac{\Delta^{4}}{q^{4}} c_{0} c_{1} c_{2}+\ldots\right]
\end{aligned}
$$


This formal expansion contains three different types of terms. The first type are the terms which contain only different combinations of $\Pi_{m}^{s}\left(q^{2}\right)$ (they are not multiplied by inverse powers of $q^{2}$ ); the third type of terms contains only different combinations of $\left(\Delta^{2} / q^{2}\right)$. The second type of terms contains the so-called mixed terms, containing the first and third types of terms in different combinations. The two last types of terms are multiplied by the corresponding powers of $1 / q^{2}$. Such structure of terms will be present in each iteration term for the full gluon form factor. However, any of the mixed terms can be split exactly into the first and third types of terms. For this purpose the formal Taylor expansions for $\Pi_{m}^{s}\left(q^{2}\right)$ around the finite subtraction point $\alpha$ should be used. Thus an exact IR structure of the full gluon form factor (which just is our primary goal to establish) is determined not only by the third type of terms. It gains contributions from the mixed terms as well, but without changing its functional dependence (see remarks below). To demonstrate this in some detail, it is convenient to express the previous expansion (A11) in terms of dimensionless variables and parameters introduced in section IV, namely

$$
z=\frac{\Delta^{2}}{q^{2}}, \quad x=\frac{q^{2}}{M^{2}}, \quad a=z x=\frac{\Delta^{2}}{M^{2}}, \quad \alpha=\frac{\mu^{2}}{M^{2}},
$$

where $M^{2}$ is some fixed mass squared, and $\mu^{2}$ is the fixed point close to $q^{2}=0$ (to be not mixed up with the tensor index). Also, in the formal PT $\Delta^{2}=0$ limit $a=0$ as well, since $M^{2}$ is fixed. On account of the relations (A12), the expansion (A11) becomes

$$
\begin{aligned}
d(x) & =\left[1-\Pi_{2}^{s}(x)+\Pi_{1}^{s}(x) \Pi_{2}^{s}(x)-\Pi_{0}^{s}(x) \Pi_{1}^{s}(x) \Pi_{2}^{s}(x)+\ldots\right] \\
& +z\left[\Pi_{2}^{s}(x) c_{1}+\Pi_{1}^{s}(x) c_{2}-\Pi_{0}^{s}(x) \Pi_{1}^{s}(x) c_{2}-\Pi_{0}^{s}(x) \Pi_{2}^{s}(x) c_{1}-\Pi_{1}^{s}(x) \Pi_{2}^{s}(x) c_{0}+\ldots\right] \\
& -z^{2}\left[\Pi_{0}^{s}(x) c_{1} c_{2}+\Pi_{1}^{s}(x) c_{0} c_{2}+\Pi_{2}^{s}(x) c_{0} c_{1}+\ldots\right] \\
& -z\left[c_{2}-\left(\frac{a}{x}\right) c_{1} c_{2}+\left(\frac{a}{x}\right)^{2} c_{0} c_{1} c_{2}+\ldots\right] .
\end{aligned}
$$

Taking into account the above-mentioned formal Taylor expansions

$$
\Pi_{m}^{s}(x)=\sum_{n=0}^{\infty}(x-\alpha)^{n} \Pi_{m}^{(n)}(\alpha)=\sum_{n=0}^{\infty}\left[\sum_{k=0}^{n} p_{n k} x^{k} \alpha^{n-k}\right] \Pi_{m}^{(n)}(\alpha),
$$

for example, the mixed term $z \Pi_{2}^{s}(x) c_{1}$ can be then exactly split/decomposed as follows:

$$
c_{1} z \Pi_{2}^{s}(x)=c_{1} z \sum_{n=0}^{\infty}\left[\sum_{k=0}^{n} p_{n k} x^{k} \alpha^{n-k}\right] \Pi_{2}^{(n)}(\alpha)=z P_{1}(\alpha)+P_{0}(\alpha)+O_{2}(x) .
$$

Here and below the dependence on all other possible parameters is not shown, for simplicity. The dimensionless function $\mathrm{O}_{2}(x)$ is of the order $x$ at small $x$; otherwise it remains arbitrary. The first term now is to be shifted to the third type of terms, while the remaining terms are to be shifted to the first type of terms. All other mixed terms of similar structure should be treated absolutely in the same way.

The mixed term $z^{2} \Pi_{0}^{s}(x) c_{1} c_{2}$ can be split as

$$
c_{1} c_{2} z^{2} \Pi_{0}^{s}(x)=c_{1} c_{2} z^{2} \sum_{n=0}^{\infty}\left[\sum_{k=0}^{n} p_{n k} x^{k} \alpha^{n-k}\right] \Pi_{0}^{(n)}(\alpha)=z^{2} P_{2}(\alpha)+z N_{1}(\alpha)+N_{0}(\alpha)+O_{0}(x),
$$

where the dimensionless function $O_{0}(x)$ is of the order $x$ at small $x$; otherwise it remains arbitrary. Again the first two terms should be shifted to the third type of terms, while the last two terms should be shifted to the first type of terms.

Similarly to the formal Taylor expansion (A14), we can write

$$
\Pi_{m}^{s}(x) \Pi_{m^{\prime}}^{s}(x)=\Pi_{m m^{\prime}}^{s}(x)=\sum_{n=0}^{\infty}(x-\alpha)^{n} \Pi_{m m^{\prime}}^{(n)}(\alpha)=\sum_{n=0}^{\infty}\left[\sum_{k=0}^{n} p_{n k} x^{k} \alpha^{n-k}\right] \Pi_{m m^{\prime}}^{(n)}(\alpha) .
$$


Then, for example the mixed term $z \Pi_{0}^{s}(x) \Pi_{1}^{s}(q x) c_{2}$ can be split as

$$
\left.c_{2} z \Pi_{0}^{s}(x) \Pi_{1}^{s}(x)=c_{2} z\right) \Pi_{01}(x)=c_{2} z \sum_{n=0}^{\infty}\left[\sum_{k=0}^{n} p_{n k} x^{k} \alpha^{n-k}\right] \Pi_{01}^{(n)}(\alpha)=z M_{1}(\alpha)+M_{0}(\alpha)+O_{01}(x),
$$

where the dimensionless function $O_{01}(x)$ is of the order $x$ at small $x$; otherwise it remains arbitrary. Again the first term should be shifted to the third type of terms, while other two terms are to be shifted to the first type of terms.

Completing this exact splitting/shifting procedure in the expansion (A13), and restoring the explicit dependence on the dimensional variable and parameters (A12), one can equivalently present the initial expansion (A11) as follows:

$$
d\left(q^{2}\right)=\left(\frac{\Delta^{2}}{q^{2}}\right) B_{1}\left(\lambda, \alpha, \xi, g^{2}, a\right)+\left(\frac{\Delta^{2}}{q^{2}}\right)^{2} B_{2}\left(\lambda, \alpha, \xi, g^{2}, a\right)+\left(\frac{\Delta^{2}}{q^{2}}\right)^{3} B_{3}\left(\lambda, \alpha, \xi, g^{2}, a\right)+\ldots+d_{3}\left(q^{2} ; \Delta^{2}\right)+\ldots,
$$

since the coefficients of the above-used expansions depend, in general, on the same set of parameters: $\lambda, \alpha, \xi, g^{2}, a$, etc. The invariant function $d_{3}\left(q^{2} ; \Delta^{2}\right)$ is dimensionless, and it is free of the power-type IR singularities; otherwise it remains arbitrary. In the formal PT $\Delta^{2}=0$ limit it survives, and is to be reduced to the sum of the first type of terms in the expansion (A11). In other words, it is a sum of $d^{M P T}\left(q^{2}\right)$ and $d^{P T}\left(q^{2}\right)$ up to third order, which have been defined in section IV. The generalization to the next iterations is almost obvious, and one finally obtains expansions (4.9)-(4.13) for the full gluon propagator.

Concluding, let us underline that the splitting/shifting procedure does not change the structure of the NL iteration solution at small $q^{2}$. It only changes the coefficients at inverse powers of $q^{2}$ in the corresponding expansion. In other words, it makes it possible to rearrange the terms in the initial expansion (A11) in order to get it in the final form (A19). Also, in the $q^{2} \rightarrow 0$ limit, it is legitimate to suppress the subtracted gluon self-energy in comparison with the mass gap term in the initial Eq. (A1). Nevertheless, as a result of the splitting/shifting procedure, which becomes almost trivial in this case, one will obtain the same expansion (A19) with only different residues, as just mentioned above. It is worth emphasizing that residues remain completely arbitrary (undetermined) in any case.

[1] W. Marciano, H. Pagels, Phys. Rep. C 36 (1978) 137.

[2] M.E. Peskin, D.V. Schroeder, An Introduction to Quantum Field Theory (AW, Advanced Book Program, 1995).

[3] A. Jaffe, E. Witten, Yang-Mills Existence and Mass Gap, http://www.claymath.org/prize-problems/, http://www.arthurjaffe.com .

[4] V. Gogokhia, Int. J. Theor. Phys. (2009) DOI: 10.1007/s10773-009-0101-3; arXiv:0806.0247 [hep-th, hep-ph].

[5] J.M. Cornwall, Phys. Rev. D 26 (1982) 1453.

[6] V.A. Rubakov, Classical Gauge Fields (Editorial YRSS, Moscaw, 1999).

[7] Quark Matter 2005, Edited by T. Csorgo, G. David, P. Levai, G. Papp (ELSEVIER, Amsterdam-...-St. Louis, 2005).

[8] M. Gyulassy, L. McLerran, arXiv:nucl-th/0405013

[9] M. Baker, Ch. Lee, Phys. Rev. D 15 (1977) 2201.

[10] C. Itzykson, J.-B. Zuber, Quantum Field Theory (Mc Graw-Hill Book Company, 1984).

[11] T. Muta, Foundations of QCD (Word Scientific, 1987).

[12] I.M. Gel'fand, G.E. Shilov, Generalized Functions, Vol. I (Academic Press, New York, 1968).

[13] G. 't Hooft, M. Veltman, Nucl. Phys. B 44 (1972) 189.

[14] V. Gogohia, Phys. Lett. B 584 (2004) 225.

[15] V. Gogohia, Phys. Lett. B 618 (2005) 103.

[16] V.G. Bornyakov, V.K. Mitrjushkin, M. Müller-Preussker, arXiv:0812.2761 [hep-lat].

[17] I.L. Bogolubsky, E.-M. Igenfritz, M. Müller-Preussker, A. Sternbeck, arXiv:0901.0736 [hep-lat].

[18] A. Cucchieri, T. Mendes, arXiv:0904.4033 [hep-lat].

[19] A. Cucchieri, T. Mendes, Phys. Rev. Lett., 100 (2008) 241601, arXiv:0712.3517] [hep-lat].

[20] A.C. Aguilar, D. Binosi, J. Papavassiliou, Phys. Rev. D 78 (2008) 025010, arXiv:0802.1870 [hep-ph].

[21] R. Alkofer, L. von Smekal, Phys. Rep. 353 (2001) 281.

[22] C.S. Fischer, A. Maas, J.H. Pawlowski, arXiv:0810.1987 [hep-ph].

[23] S.P. Sorella, arXiv:0905.1010 [hep-th].

[24] D. Dudal, J.A. Gracey, S.P. Sorella, N. Vandersickel, H. Verschelde, Phys. Rev. D 78 (2008) 065047, arXiv:0806.4348 [hep-th].

[25] D. Zwanziger, arXiv:0904.2380 [hep-th].

[26] K.-I. Kondo, arXiv:0907.3249 [hep-th]. 